\section{Slow-wave sleep takes the leading role in memory reorganization}

\section{Susanne Diekelmann and Jan Born}

We recently proposed a comprehensive framework for sleep-dependent memory consolidation suggesting that, during slowwave sleep (SWS), memory representations are transferred from a temporary to a longterm store and thereby undergo reorganization in a process of system consolidation (The memory function of sleep. Nature Rev. Neurosci. 11, 114-126 (2010)) $)^{1}$. Further, we suggested that this system consolidation process is complemented by the strengthening of synaptic connections - that is, synaptic consolidation - during the ensuing rapid eye movement (REM) sleep. Walker and Stickgold extend this framework by specifying cognitive features that are essential to the process of memory reorganization during sleep (Overnight alchemy: sleep-dependent memory evolution. Nature Rev. Neurosci. 19 Feb 2010 (doi:10.1038/nrn2762-c1)) $)^{2}$. Four processes are proposed: an initial hippocampal binding of separate neocortical memory traces, followed by the unitization, assimilation and abstraction of more generalized representations at the neocortical level. This is an elegant cognitive concept that compellingly integrates some recent findings on the effects of sleep on problem-solving tasks.

However, Walker and Stickgold's proposal $^{2}$ to link such processes of reorganization to REM sleep seems to be at some variance with the available experimental evidence. Forming part of a superordinate system consolidation process, the proposed 'evolutionary' stages of memory consolidation are more likely to take place during SWS, for three reasons. First, the reorganization of memory representations requires the reactivation of the representations. At the neuronal level, memory reactivation seems to occur most robustly during SWS $^{3-5}$ and rarely during REM sleep. Of note, reports of dreams after awakenings from REM sleep in this context are often mistaken for indicators of the occurrence of memory reactivation during REM sleep. However, such reports are generated while the brain is 'awake' and do not necessarily provide an accurate picture of the processes that actually take place during REM sleep. Second, there is considerable evidence (summarized in REF. 1) that electrophysiological phenomena linked to SWS, such as sharp wave-ripples, spindles and slow oscillations, coordinately contribute to the transfer of memory information from the hippocampus to the neocortex, a process that is thought to underlie the proposed reorganization of memory representations $s^{6,7}$. Low acetylcholinergic tone, which occurs during SWS, is a further prerequisite for such transfer ${ }^{8,9}$. Third, a direct comparison of the effects of REM-rich and SWSrich sleep has provided solid behavioural evidence that the proposed process of 'abstraction' - that is, gaining insight into hidden rules - benefits from SWS rather than REM sleep ${ }^{10}$.

All this does not exclude a contribution of REM sleep to the reorganization of memories beyond that of a synaptic strengthening of the reorganized (neocortical) representations. However, the ultimate contributions of REM sleep to memory consolidation have so far remained enigmatic ${ }^{11}$. Given the many proposals for the role of REM sleep in memory, it seems that the time is ripe to subject some of these proposals to systematic scrutiny.

Susanne Diekelmann and Jan Born are at the University of Lübeck, Department of Neuroendocrinology, Haus 50, 2. OG, Ratzeburger Allee 160, 23538 Lübeck, Germany. Correspondence to J. B. e-mail:born@kfg.uni-luebeck.de doi:10.1038/nrn2762-c2

1. Diekelmann, S. \& Born, J. The memory function of sleep. Nature Rev. Neurosci. 11, 114-126 (2010). 2. Walker, M. P. \& Stickgold, R. Overnight alchemy: sleep-dependent memory evolution. Nature Rev. Neurosci. 19 Feb 2010 (doi:10.1038/nrn2762-c1).

3. Wilson, M. A. \& McNaughton, B. L. Reactivation of hippocampal ensemble memories during sleep. Science 265, 676-679 (1994).

4. Nadasdy, Z., Hirase, H., Czurko, A., Csicsvari, J. \& Buzsaki, G. Replay and time compression of recurring spike sequences in the hippocampus. J. Neurosci. 19 9497-9507 (1999).

5. Ribeiro, S. et al. Long-lasting novelty-induced neuronal reverberation during slow-wave sleep in multiple forebrain areas. PLoS Biol. 2, E24 (2004).

6. Sirota, A., Csicsvari, J., Buhl, D. \& Buzsaki, G. Communication between neocortex and hippocampus during sleep in rodents. Proc. Natl Acad. Sci. USA 100, 2065-2069 (2003).

7. Steriade, M. Grouping of brain rhythms in corticothalamic systems. Neuroscience 137 1087-1106 (2006)

8. Hasselmo, M. E. Neuromodulation: acetylcholine and memory consolidation. Trends Cogn. Sci. 3, 351-359 (1999).

9. Gais, S. \& Born, J. Low acetylcholine during slow-wave sleep is critical for declarative memory consolidation. Proc. Natl Acad. Sci. USA 101, 2140-2144 (2004).

10. Yordanova, J. et al. Shifting from implicit to explicit knowledge: different roles of early- and late-night sleep. Learn. Mem. 15, 508-515 (2008).

11. Siegel, J. M. The REM sleep-memory consolidation hypothesis. Science 294, 1058-1063 (2001). 